\title{
Enhancement of Absorption and Color Contrast in Ultra-Thin Highly Absorbing Optical Coatings
}

\section{Citation}

Kats, Mikhail A., Steven J. Byrnes, Romain Blanchard, Mathias Kolle, Patrice Genevet, Joanna Aizenberg, and Federico Capasso. 2013. "Enhancement of Absorption and Color Contrast in Ultra-Thin Highly Absorbing Optical Coatings." Applied Physics Letters 103 (10): 101104. https:// doi.org/10.1063/1.4820147.

\section{Permanent link}

http://nrs.harvard.edu/urn-3:HUL.InstRepos:37933105

\section{Terms of Use}

This article was downloaded from Harvard University's DASH repository, and is made available under the terms and conditions applicable to Other Posted Material, as set forth at http:// nrs.harvard.edu/urn-3:HUL.InstRepos:dash.current.terms-of-use\#LAA

\section{Share Your Story}

The Harvard community has made this article openly available.

Please share how this access benefits you. Submit a story.

Accessibility 


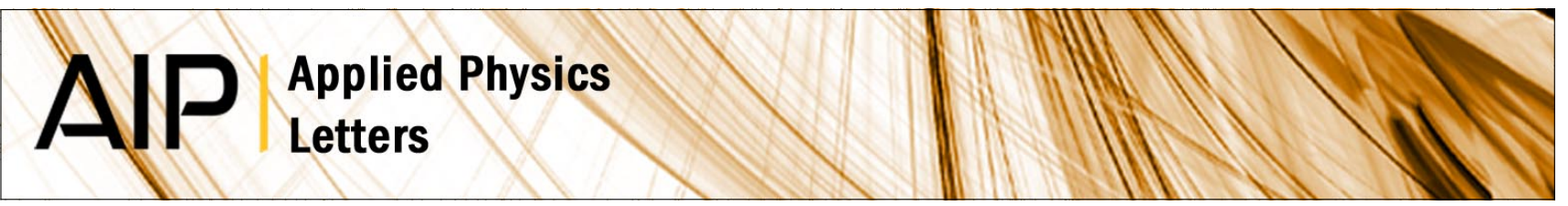

\section{Enhancement of absorption and color contrast in ultra-thin highly absorbing optical coatings}

Mikhail A. Kats, Steven J. Byrnes, Romain Blanchard, Mathias Kolle, Patrice Genevet et al.

Citation: Appl. Phys. Lett. 103, 101104 (2013); doi: 10.1063/1.4820147

View online: http://dx.doi.org/10.1063/1.4820147

View Table of Contents: http://apl.aip.org/resource/1/APPLAB/v103/i10

Published by the AIP Publishing LLC.

\section{Additional information on Appl. Phys. Lett.}

Journal Homepage: http://apl.aip.org/

Journal Information: http://apl.aip.org/about/about_the_journal

Top downloads: http://apl.aip.org/features/most_downloaded

Information for Authors: http://apl.aip.org/authors

\section{ADVERTISEMENT}

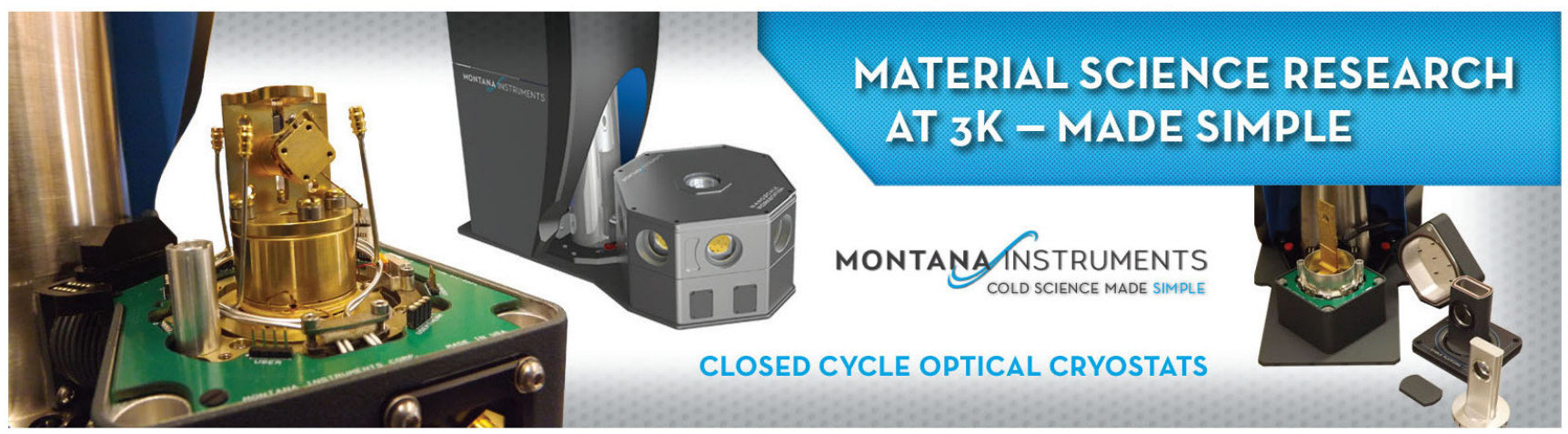




\title{
Enhancement of absorption and color contrast in ultra-thin highly absorbing optical coatings
}

\author{
Mikhail A. Kats, ${ }^{\text {a) }}$ Steven J. Byrnes, ${ }^{\text {a) }}$ Romain Blanchard, ${ }^{\text {a) }}$ Mathias Kolle, Patrice Genevet, \\ Joanna Aizenberg, and Federico Capasso b) \\ School of Engineering and Applied Sciences, Harvard University, Cambridge, Massachusetts 02138, USA
}

(Received 23 June 2013; accepted 19 August 2013; published online 4 September 2013)

\begin{abstract}
Recently a new class of optical interference coatings was introduced which comprises ultra-thin, highly absorbing dielectric layers on metal substrates. We show that these lossy coatings can be augmented by an additional transparent subwavelength layer. We fabricated a sample comprising a gold substrate, an ultra-thin film of germanium with a thickness gradient, and several alumina films. The experimental reflectivity spectra showed that the additional alumina layer increases the color range that can be obtained, in agreement with calculations. More generally, this transparent layer can be used to enhance optical absorption, protect against erosion, or as a transparent electrode for optoelectronic devices. (C) 2013 AIP Publishing LLC. [http://dx.doi.org/10.1063/1.4820147]
\end{abstract}

Optical interference coatings comprising multiple layers of transparent materials are a cornerstone of modern optical technology. ${ }^{1}$ These coatings utilize the principle of thin film interference to enable effects such as anti-reflection (AR) and high-reflection (HR), the former being especially important for imaging equipment such as microscope objectives, telephoto lenses, and prescription glasses.

Sophisticated optical coating design strategies often utilize many films of varying materials and thicknesses to achieve particular performance benchmarks, with architectures that are created and optimized using computer software. $^{2}$ The simplest and most well-known conventional optical coating is the quarter-wave AR coating, in which the reflectivity at the interface between two dielectric media with refractive indices $n_{1}$ and $n_{3}$ is minimized by inserting a dielectric film with refractive index $n_{2}$ and thickness $\lambda / 4 n_{2}$ where $n_{3}>n_{2}>n_{1}$ in between ( $\lambda$ is the free-space wavelength).

By relaxing the constraint that the materials comprising the substrate and the thin films be purely dielectric (i.e., transparent and lossless), coatings that minimize reflectivity of a surface can be made thinner than the standard quarter-wave dimension. One example is anodized titanium: by electrochemically forming thin layers of transparent titanium oxide on a titanium substrate, a variety of colors can be generated which are a result of wavelength-selective absorption. ${ }^{3}$ Since the oxide is transparent, the optical absorption occurs upon repeated reflections from the oxide/titanium interface. In anodized titanium, light can be efficiently absorbed given layers that are approximately between $\lambda /\left(6 n_{\mathrm{TiO} 2}\right)$ and $\lambda /\left(5 n_{\mathrm{TiO} 2}\right)$, where $n_{\mathrm{TiO} 2}$ is the refractive index of the oxide; the reason that the thickness can be smaller than a quarter-wavelength is that the phase shift upon reflection at the oxide/titanium interface differs from $\pi$ because the real and imaginary parts of the complex refractive index of titanium are comparable and both not much larger than the index of the oxide (e.g., at $\lambda=600 \mathrm{~nm}, n_{T i} \simeq 2.6+3.7 i$ ).

Recently, we demonstrated that strong optical thin-film interference effects can be observed even for highly lossy

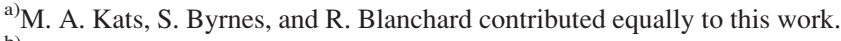
b) capasso@seas.harvard.edu
}

dielectric films. ${ }^{4,5}$ By combining metals or metal-like materials with dielectrics featuring strong optical absorption, the thickness of the films can be reduced even further (e.g. $\sim \lambda /(20 n)$ ) due to the nontrivial (not 0 or $\pi$ ) reflection phase shifts at interfaces between one or more lossy materials. These reflection phase shifts can "make up" for the propagation phase shift that would be accumulated in thicker films, leading to dramatically enhanced absorption in the already-lossy dielectric. This concept of an "ultra-thin film resonance" is applicable to a variety of spectral ranges and applications: to date it has been used in the visible for coloring metals ${ }^{4}$ or solar energy harvesting, ${ }^{6,7}$ and in the infrared for static ${ }^{8}$ or dynamic ${ }^{5,9}$ modulation of absorption and thermal emission. In general, high efficiency optical absorption across a large bandwidth within films of subwavelength thickness is promising for a variety of light harvesting, detection, and imaging applications

The functionality of deep subwavelength optical interference coatings and absorbers can be augmented by integration of transparent layers on top of the lossy layers. These can be used as protective coatings for harsh environments, as transparent electrodes for optoelectronic devices, or to modify the optical interference conditions. Here, we show that an additional sub-quarter-wave layer of a transparent dielectric can be used as another degree of freedom in tailoring the absorption within ultra-thin, lossy layers. We demonstrate that the addition of this layer enhances the color contrast of color coatings comprising nanometer-thick films of amorphous germanium $(\mathrm{Ge})$ on gold $(\mathrm{Au})$ substrates.

In Ref. 4, we used uniform evaporated films of amorphous $\mathrm{Ge}$ of thickness between $5 \mathrm{~nm}$ and $25 \mathrm{~nm}$ on an optically-thick gold substrate to obtain colors including shades of pink, violet, and blue (Fig. 1(a)). In this work we used sputtering to deposit an additional sub-quarter-wave layer of aluminum oxide $\left(\mathrm{Al}_{2} \mathrm{O}_{3}\right)$ on top of the Ge coatings to both protect the coatings and alter the reflectivity (Fig. 1(b)).

To limit the number of depositions as well as to demonstrate the full range of possible configurations of the $\mathrm{Au} / \mathrm{Ge}$ geometry, we developed a deposition method which forms a thickness gradient of the Ge film on an Au substrate. This allows us to have access to all thicknesses of $\mathrm{Ge}$ on a single 
(a)

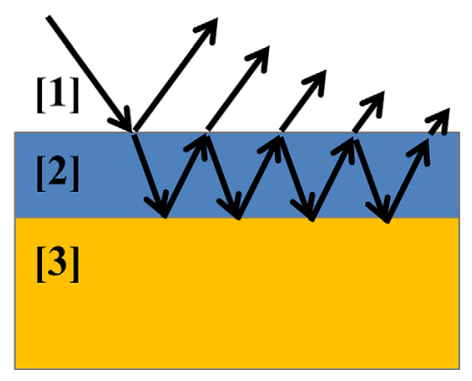

(b)

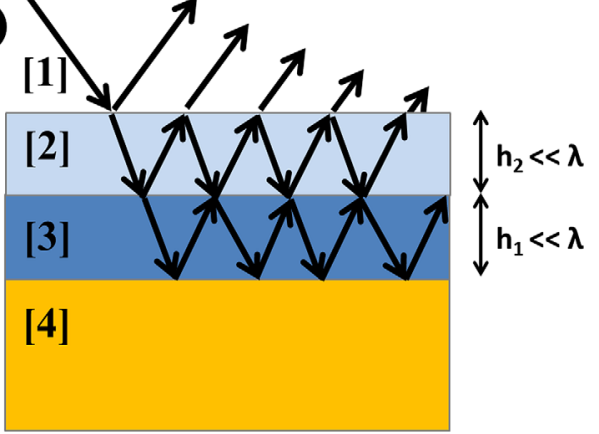

FIG. 1. (a) Schematic of the thin film geometry used in Ref. 4 and as the basis of the present work. A continuous film of amorphous germanium (Ge, shown as dark blue, thickness $h_{1}$ ) of subwavelength thickness is deposited over a gold $(\mathrm{Au})$ substrate, and the reflectivity is probed from the top. (b). A subwavelength layer of transparent alumina $\left(\mathrm{Al}_{2} \mathrm{O}_{3}\right.$, shown as light blue, thickness $\mathrm{h}_{2}$ ) is deposited over the structure in (a). sample. After coating a section of silicon wafer with an optically thick $(\sim 150 \mathrm{~nm})$ layer of $\mathrm{Au}$, we inserted the sample $\mathrm{Au}$-side-down into an electron beam evaporator for deposition of amorphous Ge. We modified the path between the crucible and the sample by introducing a slit aperture into the chamber (Fig. 2). The aperture changes the line-of-sight between the crucible and the sample so that the middle of the sample "sees" a large portion of the crucible, while points farther away from the middle of the sample see progressively less of the crucible. As a result, the deposition rate varies across the sample, and a gradient of thickness can be generated. The distances between the crucible and the aperture and between the aperture and the sample were $16 \mathrm{~cm}$ and $15.2 \mathrm{~cm}$, respectively, the width of the slit aperture was $8 \mathrm{~mm}$ wide, and the melted Ge in the crucible is approximately circular with a diameter of $2 \mathrm{~cm}$. While the dynamics of the deposition are beyond the scope of this work, back of the envelope calculations indicate that the resulting thickness profile is roughly linear.

We performed three depositions of $\mathrm{Al}_{2} \mathrm{O}_{3}$ over the top of this sample, each time masking off a portion of it, to create a sample which comprises a continuum of thickness of $\mathrm{Ge}$ and several different thicknesses of $\mathrm{Al}_{2} \mathrm{O}_{3}$. The depositions were performed using a magnetron sputterer with an $\mathrm{Al}_{2} \mathrm{O}_{3}$ target. A photo of the resulting sample is shown in Fig. 3(a), using standard fluorescent ceiling lights for illumination and no additional postprocessing. The thickness of $\mathrm{Al}_{2} \mathrm{O}_{3}$ was determined using a scanning ellipsometer, assuming $n_{A / 2 O 3}=1.7$. Note that even the thickest of the $\mathrm{Al}_{2} \mathrm{O}_{3}$ films is still significantly thinner than a quarter-wavelength across the entire visible spectrum. Fig. 3(a) immediately shows how the deposition of the $\mathrm{Al}_{2} \mathrm{O}_{3}$ layers affects the optical properties of the $\mathrm{Au} / \mathrm{Ge}$ coatings. The interference colors in region (i) match those obtained in Ref. 4, though here the entire gradient of colors possible with this particular set of materials is visible. This color gradient persists in all four sections, but the color contrast across the gradient increases significantly for regions (iii) and (iv) compared to region (i) which has no $\mathrm{Al}_{2} \mathrm{O}_{3}$ coating.

We performed a set of analytical calculations to obtain the theoretical reflectivity and color values corresponding to our fabricated sample. We utilized the transfer matrix formalism $^{10}$ to obtain the angle- and wavelength-dependent reflectivities of the multi-layer geometries, using the complex refractive index values of evaporated $\mathrm{Ge}$ and $\mathrm{Au}$ as measured with spectroscopic ellipsometry. ${ }^{4}$ Note that the thicknesses of the $\mathrm{Ge}$ and $\mathrm{Al}_{2} \mathrm{O}_{3}$ films are varying across our sample (Fig. $3(a)$ ), so every point on the sample has its own reflectivity spectrum. Following these calculations, we rendered an image that corresponds to the photograph in Fig. 3(a).

We used the ColorPy package ${ }^{11}$ to convert each reflectivity spectrum into the corresponding standard red-greenblue (RGB) color values. The process involves integrating the reflectivity spectrum with three standard "matching functions" as defined by the International Commission on Illumination in 1931 (CIE 1931), which accounts for the response of the three different photoreceptors in the eye. Using the "D65" standard illuminant, which approximates normal daylight, RGB values for a particular reflectivity spectrum are generated. The generated image is shown in Fig. 3(b), oriented so that it corresponds to the photograph in (a)

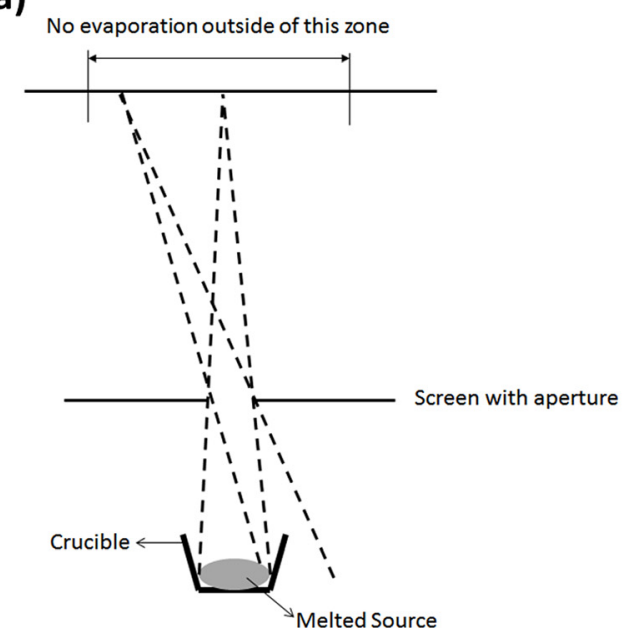

(b)

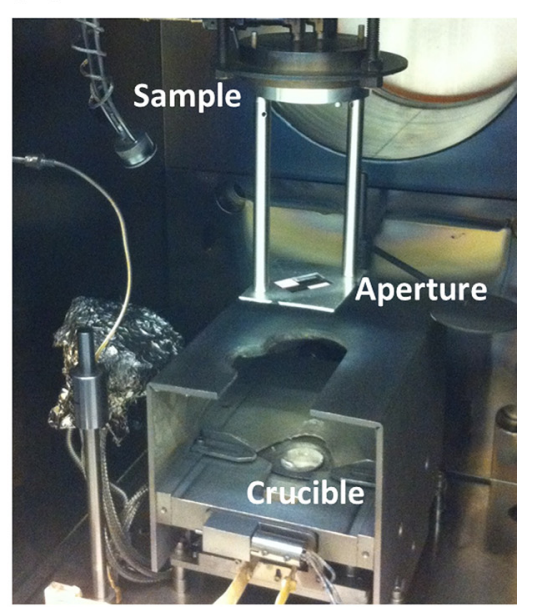

FIG. 2. Schematic (a) and photograph (b) of our evaporator. A rectangular aperture is placed in the evaporation path between the crucible and sample. $\mathrm{Ge}$ was deposited onto a gold substrate, creating an amorphous Ge film with a thickness gradient. 
Fig. 3(a). This image was generated for a $30^{\circ}$ angle of incidence which roughly corresponds to the angle at which the photo in Fig. 3(a) was taken, and prior to color rendering the s- and p-polarized spectra were averaged to simulate unpolarized light.

The values obtained from integrating a spectrum with the three CIE 1931 matching functions can be converted into two parameters: "brightness" (or "luminance"), and a twodimensional "chromaticity" value usually written as $(\mathrm{x}, \mathrm{y}){ }^{12}$ The chromaticity value can also be converted into "hue" and "saturation" values. We plotted the generated colors on a chromaticity map shown in Fig. 3(c), where the four curves correspond to the four sections in Figs. 3(a) and 3(b), and moving along the curve clockwise corresponds to increasing Ge thickness. For increasing $\mathrm{Al}_{2} \mathrm{O}_{3}$ thickness, the curves cover a larger portion of the $\mathrm{x}-\mathrm{y}$ chromaticity space compared to no $\mathrm{Al}_{2} \mathrm{O}_{3}$, demonstrating quantitatively the increase in color contrast which is apparent from Figs. 3(a) and 3(b).

To perform a more careful comparison between our experimental and theoretical results, we measured the
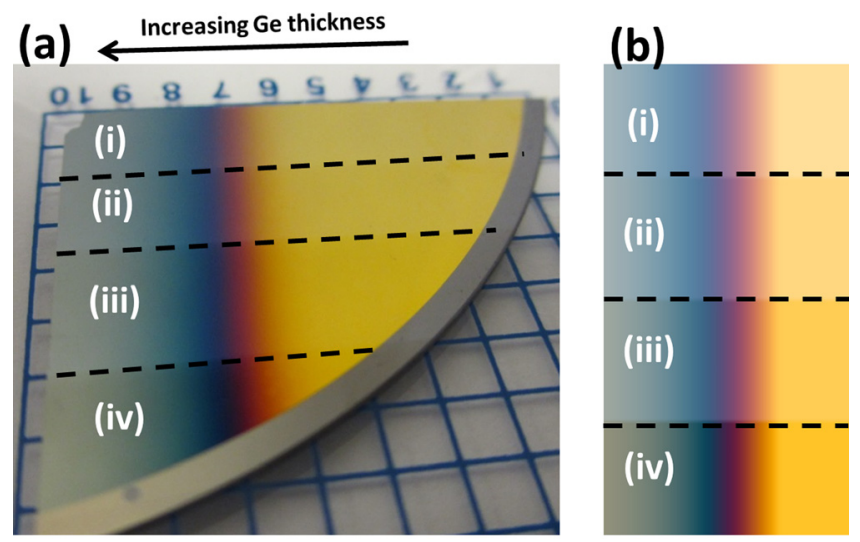

(c)

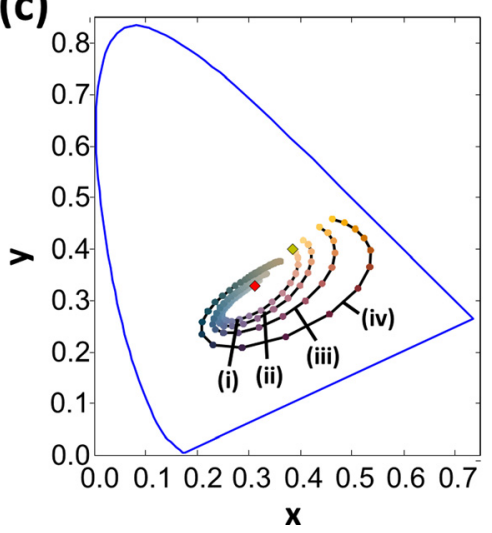

FIG. 3. (a) Photo of the sample comprising optically-thick Au deposited on a silicon wafer, with a film of Ge of gradually varying thickness, overcoated with different layers of $\mathrm{Al}_{2} \mathrm{O}_{3}$. The four sections (i)-(iv) indicate areas where no $\mathrm{Al}_{2} \mathrm{O}_{3}$ was deposited, or areas with $14 \mathrm{~nm}, 28 \mathrm{~nm}$, and $44 \mathrm{~nm}$ of $\mathrm{Al}_{2} \mathrm{O}_{3}$, respectively. (b) Simulated image corresponding to the photo in (a), with the calculated reflectivities of each section obtained using complex refractive index values of $\mathrm{Au}$ and Ge from Ref. 4 and assuming $n_{A 12 O 3}=1.7$, and then converted into RGB colors. The calculation was done for unpolarized light at a $30^{\circ}$ angle of incidence to match the photograph. (c) Chromaticity diagram corresponding to the image in (b), showing that for increasing $\mathrm{Al}_{2} \mathrm{O}_{3}$ thickness, the Ge gradient sweeps out a bigger area in the $\mathrm{x}-\mathrm{y}$ chromaticity space. The four trajectories (i)-(iv) correspond to the four $\mathrm{Al}_{2} \mathrm{O}_{3}$ thicknesses, and each point corresponds to a certain thickness of Ge. The blue "shark fin" denotes the set of the most "saturated" or "pure" colors that can be defined. reflectivity spectrum at various points on the sample. We used an optical microscope with a $5 \times, 0.15 \mathrm{NA}$ objective and a halogen light to illuminate the sample, and used an additional port to confocally collect a portion of the reflected light into a multimode optical fiber $(400 \mu \mathrm{m}$ core), which was then sent to a grating spectrometer (Ocean Optics). The collection spot was $80 \mu \mathrm{m}$ in diameter, which is much smaller than the spatial extent of our Ge thickness gradient, and therefore we assumed that the thickness change was negligible over the spot. We performed background subtraction and normalization to a flat silver mirror (reflectance $\geq 0.95$ ). Given our 0.15 NA objective, the incoming and collected light had a variation of incidence angles from $0^{\circ}$ to $8.6^{\circ}$, which we assume to be negligible because of the relatively weak dependence of the reflectivity of ultra-thin films to the angle of incidence. ${ }^{4}$ The spectra were recorded with a wavelength resolution of $\sim 0.35 \mathrm{~nm}$, and the data were smoothed to reduce spectrometer noise. We used an automated, twoaxis translation stage to scan across the sample, acquiring spectra at $22 \times 40=880$ points with a spacing of $500 \mu \mathrm{m}$ along the direction of the gradient and $600 \mu \mathrm{m}$ along the perpendicular direction.

The experimental spectra are summarized in Figs. 4(a)-4(d). Four reflectivity curves are shown per panel, corresponding to no $\mathrm{Al}_{2} \mathrm{O}_{3}$ film (solid line), and $\mathrm{Al}_{2} \mathrm{O}_{3}$ films with a thickness of $14 \mathrm{~nm}$ (dashed), $28 \mathrm{~nm}$ (dashed-dotted), and $44 \mathrm{~nm}$ (dotted). Panels (a)-(d) correspond to Ge film thicknesses of approximately $3 \mathrm{~nm}, 7.5 \mathrm{~nm}, 12 \mathrm{~nm}$, and $15 \mathrm{~nm}$, respectively. These thickness values were determined by comparison to the transfer matrix calculations (Figs. 4(e)-4(h)) which are in good agreement with the experiment. The differences between the regions with different $\mathrm{Al}_{2} \mathrm{O}_{3}$ films are clear from both the experimental and calculated spectra: the $\mathrm{Al}_{2} \mathrm{O}_{3}$ layer serves to increase the absorption in the Ge layer, reducing the reflectivity (note that the $\mathrm{Al}_{2} \mathrm{O}_{3}$ layer itself has negligible optical losses), and this effect becomes stronger with increasing $\mathrm{Al}_{2} \mathrm{O}_{3}$ thickness. Very thin $\mathrm{Al}_{2} \mathrm{O}_{3}$ layers do not substantially perturb the reflectivity, and it is likely that sub- $10 \mathrm{~nm}$ layers will be nearly imperceptible. As the thickness increases, the $\mathrm{Al}_{2} \mathrm{O}_{3}$ layer begins to operate more and more like an AR coating, even though the thicknesses that we have studied here do not reach a quarter wavelength anywhere in the visible spectrum (at the shortest wavelengths $(\lambda=450 \mathrm{~nm})$, the $44 \mathrm{~nm} \mathrm{Al}_{2} \mathrm{O}_{3}$ film corresponds to approximately $\left.\lambda /\left(6 n_{A 12 O 3}\right)\right)$. Note that because the optical phase shift upon reflection from the $\mathrm{Al}_{2} \mathrm{O}_{3} / \mathrm{Ge}$ interface is not $\pi$ as it would have been if the Ge were lossless, the $\mathrm{Al}_{2} \mathrm{O}_{3}$ thickness that minimizes reflection is not necessarily $\lambda /\left(4 n_{A 12 O 3}\right)$, and varies significantly as a function of wavelength because of the large dispersion of $\mathrm{Ge}$ and the underlying $\mathrm{Au}$. Our calculations show that a thickness of roughly $\lambda /$ $\left(5 n_{A 12 O 3}\right)$ to $\lambda /\left(7 n_{A l 2 O 3}\right)$ is needed to minimize the reflectivity at various wavelengths within the visible range.

We note that the material choice for highly lossy optical coatings is not necessarily limited to semiconductors or other homogeneous materials. Various lossy thin-film composite structures comprising two or more different materials that can be described by some effective complex refractive index have been proposed and investigated, including core/shell nanoparticle arrays, ${ }^{13}$ metal/air subwavelength gratings, ${ }^{14}$ 
Experiment
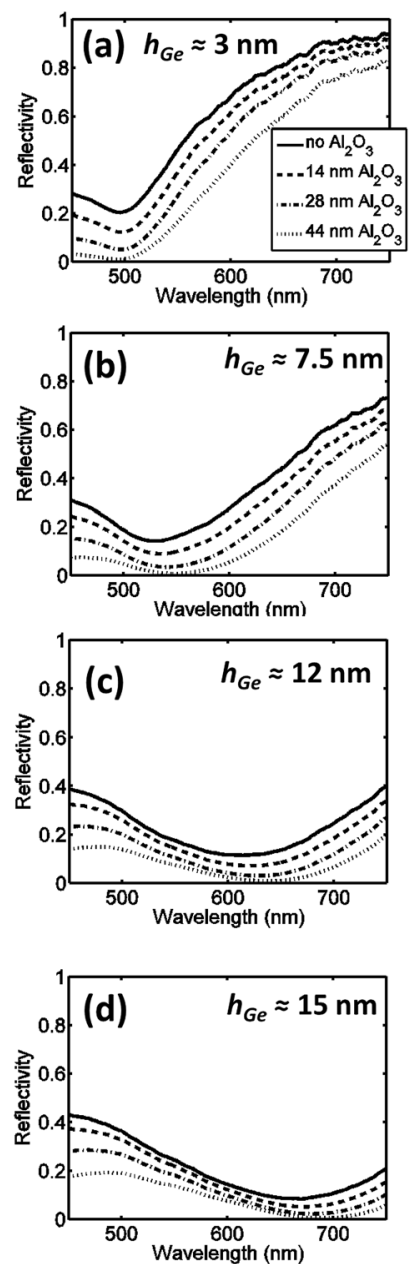

Simulation
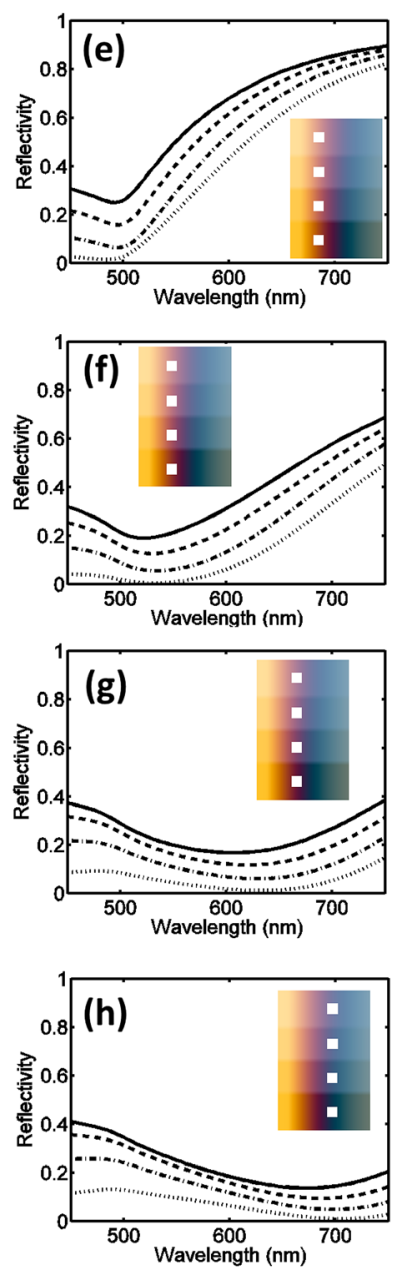

FIG. 4. Measured (a-d) and calculated (e-h) reflectivity spectra for normal incidence at positions on the sample corresponding to four different values of Ge thickness (approximately $3 \mathrm{~nm}, 7.5 \mathrm{~nm}, 12 \mathrm{~nm}$, and $15 \mathrm{~nm}$, respectively) and four different values of $\mathrm{Al}_{2} \mathrm{O}_{3}$ thickness $(0$, solid line; $14 \mathrm{~nm}$, dashed; $28 \mathrm{~nm}$, dashed-dotted; $44 \mathrm{~nm}$, dotted). The insets of (e-h) represent calculated images of the sample corresponding to the picture in Fig. 3(b), except recalculated for normal incidence instead of a $30^{\circ}$ incidence angle (as in Fig. 3(b)). The white squares in the insets indicate the positions where the four reflectivity curves are calculated. The approximate thickness of the Ge film at these locations are shown in the insets of $(a-d)$. Note that because the gold films are opaque, the reflectivity $R=1-A$, where $A$ is the absorptivity.

split ring resonator metamaterials, ${ }^{15}$ and others. Compared to the use of homogeneous materials as in the present work these approaches offer additional design flexibility but at the cost of significantly more involved fabrication which often requires high resolution lithography.
Recently demonstrated ultra-thin optical coatings comprising metals and dielectrics with a large degree of optical loss show promise for colorizing metals and for a variety of device applications such as photodetection, energy harvesting, and engineering of thermal emission. Understanding the effect of additional transparent layers deposited over these coatings is important, because such layers can be used as protection against chemical or physical erosion or as transparent electrical contacts for optoelectronic devices. In this work, we showed that depositing transparent layers of alumina on top of ultra-thin coatings comprising gold and germanium can enhance the optical absorption as well as the color range that can be attained.

We acknowledge the financial support from the Air Force Office of Scientific Research under grant numbers FA9550-12-1-0289 and FA9550-09-1-0669-DOD35CAP. M. Kats was supported by the National Science Foundation through a Graduate Research Fellowship. M. Kolle was supported by the Alexander-von-Humboldt Foundation. The thin film depositions were performed at the Harvard Center for Nanoscale Systems, which is a member of the National Nanotechnology Infrastructure Network.

${ }^{1}$ H. A. Macleod, Thin-Film Optical Filters (Adam Hilger Ltd, Bristol, 1986).

${ }^{2}$ A variety of commercial software for thin film calculation and optimization is available, including Essential Macleod, FilmStar, Film Wizard, and OptiLayer.

${ }^{3}$ E. Gaul, J. Chem. Educ. 70, 176 (1993).

${ }^{4}$ M. A. Kats, R. Blanchard, P. Genevet, and F. Capasso, Nature Mater. 12, 20 (2013).

${ }^{5}$ M. A. Kats, D. Sharma, J. Lin, P. Genevet, R. Blanchard, Z. Yang, M. M. Qazilbash, D. N. Basov, S. Ramanathan, and F. Capasso, Appl. Phys. Lett. 101, 221101 (2012).

${ }^{6}$ H. Dotan, O. Kfir, E. Sharlin, O. Blanck, M. Gross, I. Dumchin, G. Ankonina, and A. Rothschild, Nature Mater. 12, 158 (2013).

${ }^{7}$ X. L. Zhang, J. F. Song, X. B. Li, J. Feng, and H. B. Sun, Appl. Phys. Lett. 102, 103901 (2013).

${ }^{8}$ W. Streyer, S. Law, G. Rooney, T. Jacobs, and D. Wasserman, Opt. Express 21, 9113 (2013).

${ }^{9}$ M. A. Kats, R. Blanchard, S. Zhang, P. Genevet, C. Ko, S. Ramanathan, and F. Capasso, e-print arXiv:1305.0033 (2013).

${ }^{10} \mathrm{P}$. Yeh, Optical Waves in Layered Media (Wiley, New Jersey, 2005).

${ }^{11}$ M. Kness, "ColorPy-A Python package for handling physical descriptions of color and light spectra," MarkKness.net, accessed on 08/06/2013 at http://markkness.net/colorpy/ColorPy.html.

${ }^{12}$ N. Ohta and A. R. Robertson, Colorimetry (Wiley, West Sussex, 2005).

${ }^{13}$ C. Hagglund, S. P. Apell, and B. Kasemo, Nano Lett. 10, 3135 (2010).

${ }^{14}$ V. G. Kravets, F. Schedin, and A. N. Grigorenko, Phys. Rev. B 78, 205405 (2008).

${ }^{15}$ N. I. Landy, S. Sajuyigbe, J. J. Mock, D. R. Smith, and W. J. Padilla, Phys. Rev. Lett. 100, 207402 (2008). 